\title{
Kastrationsresistentes Prostatakarzinom: Erstlinientherapie nicht zu früh abbrechen
}

- Patienten mit metastasiertem, kastrationsresistentem Prostatakarzinom (mCRPC) sprechen in der Regel gut auf eine Erstlinienchemotherapie an. Unabhängig vom Ansprechen wird jedoch früher oder später jeder Patient progredient. Man müsse daher schon mit Therapiebeginn überlegen, welche Second-Line-Optionen bestehen, betonte Prof. Axel Heidenreich, Aachen. So könne man anhand von Parametern wie Toxizität oder Metastasierung schon frühzeitig einschätzen, in welchem Ausmaß man dem Patienten eine Zweitlinientherapie zumuten kann.

Bei Patienten, die zunächst gut auf die First-Line-Behandlung angesprochen haben und noch über eine ausreichende Knochenmarkreserve verfügen, bietet sich eine repetitive Chemotherapie an.

Eine weitere Therapieoption könnte künftig im neuartigen Taxan Cabazitaxel bestehen. Heidenreich präsentierte Daten der multizentrischen, randomisierten Phase-III-Stu- die TROPIC mit 755 Patienten, die zuvor mit Docetaxel behandelt worden waren. Die Patienten erhielten dreiwöchentlich entweder Cabazitaxel $25 \mathrm{mg} / \mathrm{m}^{2}$ in Kombination mit $10 \mathrm{mg} / \mathrm{d}$ Prednison (CBZP) oder Mitoxantron $12 \mathrm{mg} / \mathrm{m}^{2}$ plus Prednison (MP). Das mittlere Gesamtüberleben betrug in der CBZP-Gruppe 15,1 Monate vs. 12,7 Monate im MP-Arm ( $p<0,0001)$, das progressionsfreie Überleben war ebenfalls signifikant verlängert (2,8 vs. 1,4 Monate, $p<0,0001$ ). Cabazitaxel war Mitoxantron in allen Subgruppen überlegen, außer wenn Patienten weniger als drei Docetaxel-Zyklen à $75 \mathrm{mg} / \mathrm{m}^{2}$ (Gesamtdosis $<225 \mathrm{mg} / \mathrm{m}^{2}$ ) erhielten. Heidenreich vermutet, dass bei diesen Patienten die Docetaxel-Therapie zu früh abgebrochen wurde, weil man einen PSA-Anstieg fälschlicherweise als Progress gewertet hatte. ,Man muss die Erstlinientherapie mit Docetaxel ausreizen, um sichergehen zu können, dass die Patienten eine DocetaxelResistenz haben und von der neuen Medi- kation profitieren, “stellte Heidenreich klar. Wichtig sei zudem bei der Cabazitaxel-Therapie, Leberfunktion und hämatotoxische Nebenwirkungen zu beobachten.

Als dritte Behandlungsoption stellte Heidenreich die metronomische Chemotherapie $(M C)$ vor, die sich für Patienten mit fehlenden Knochenmarkreserven, schlechter Leberfunktion oder frühem Rezidiv unter Docetaxel eignet. Ein mögliches Therapieregime ist die niedrigdosierte, kontinuierliche, pausenfreie Gabe von Etoposid $50 \mathrm{mg} / \mathrm{m}^{2}$, Estramustinphosphat $3 \times 280 \mathrm{mg} / \mathrm{d}$ und Cyclophosphamid $100 \mathrm{mg} / \mathrm{m}^{2}$. Sinkt der PSA-Wert nach drei vierwöchigen Zyklen nicht, sollte die Therapie bis zum Progress fortgesetzt werden.

slx

Fach-Pressekonferenz „Aktuelle und zukünftige Behandlungsstrategien beim MCRPC“ im Rahmen des 62. DGU-Kongresses, Düsseldorf, 23. September 2010. Veranstalter: Sanofi-Aventis, Frankfurt am Main

\section{Galenus-Preis für Innovationen und Grundlagenforschung verliehen}



_ In diesem Jahr hatte es die Galenus-Jury nicht leicht, sich für jeweils einen Gewinner des von der Ärzte Zeitung (Springer Medizin) gestifteten Preises in den Kategorien „Primary Care“, „Specialist Care“ sowie „Grundlagenforschung“ zu entscheiden. So sei die Entscheidung auch nicht einstimmig wie im vergangenen Jahr gefallen, ,,aber nach langem hin und her-doch mit über- wältigender Mehrheit", so Prof. Erland Erdmann, Köln, Vorsitzender der GalenusJury bei der Verleihung des Preises.

Removab $^{\circledR}$ (Catumaxomab) von Fresenius Biotech - der Gewinner in der Kategorie „Specialist Care“ - erfülle die Forderung, die der französische Pharmakologe und Medizinjournalist Roland Mehl an eine Arznei stellte, als er die Auszeichnung für pharmakologische Innovationen 1970 ins Leben rief, so Erdmann: Sie müsse einen neuen Wirkmechanismus haben und die Lebensqualität erhöhen. Beides treffe auch auf Catumaxomab zu. Das Präparat ist das erste zugelassene Arzneimittel zur Behandlung von Patienten mit malignem Aszites. Als Gewinner in der Kategorie „Primary Care" wählte die Jury den neuartigen direkten Thrombinhemmer Pradaxa ${ }^{\circledR}$ (Dabigatranetexilat) von Boehringer Ingelheim. Mit dem Medikament lassen sich bei Patienten mit Vorhofflimmern Schlaganfälle verhindern. Vorteil von Dabigatran sei, dass Einschränkungen wie bei der Therapie mit Marcumar entfielen. Die Zulassung des
Präparates in Europa zur Schlaganfallprophylaxe bei Vorhofflimmern werde in wenigen Monaten erwartet.

Dr. Kristina Lorenz vom Institut für Pharmakologie und Toxikologie an der Uni Würzburg wurde für ihre Forschung zur Entstehung der krankhaften Herzhypertrophie ausgezeichnet. Lorenz erhält den Preis für die Aufklärung eines komplexen molekularen Mechanismus, in dessen Folge es zur krankhaften Herzvergrößerung kommt. Diese Entdeckungen seien zudem interessant für die Onkologie, weil die Hemmstoffe auch antiproliferativ wirken und damit therapeutisch gegen Krebs genutzt werden könnten.

Den Charity-Award erhält in diesem Jahr die Deutsche Knochenmarkspenderdatei (DKMS). Die 1991 ins Leben gerufene DKMS ist mit knapp 2,2 Millionen registrierten potenziellen Spendern die größte Stammzellspenderdatei der Welt.

ple

Gala „Galenus-von-Pergamon-Preis“, 22. Oktober 2010, Berlin. Veranstalter: Springer Medizin, Neu-Isenburg 